\title{
Multidrug-Resistant Gram-Negative Pathogens: Multiple Approaches and Measures for Prevention
}

\author{
Jan E. Patterson, MD
}

Antimicrobial resistance continues to emerge in our hospitals. Despite the predominance of gram-positive healthcare-associated infections in the past 2 decades, gram-negative pathogens continue to be a concern because of the high mortality from these infections among seriously ill patients. ${ }^{1,2}$ Although several alternative antimicrobials active against multidrugresistant gram-positive pathogens are now available, multidrug resistant gram-negative pathogens continue to emerge and new alternative therapies are not available. Preventive measures, then, become all the more important. Pseudomonas aeruginosa, Klebsiella pneumoniae, and Enterobacter species are among the most problematic gram-negative pathogens with emerging resistance. Four articles in this month's journal address important issues in the prevention of the emergence of resistance in these pathogens.

Lautenbach et al. ${ }^{3}$ and Fortaleza et al. ${ }^{4}$ study risk factors associated with imipenem resistance ${ }^{3,4}$ and ceftazidime resistance ${ }^{3}$ in $P$. aeruginosa. The setting of the study by Fortaleza et al. ${ }^{4}$ was a 400 -bed general teaching hospital in Campinas, Brazil. Cases in which imipenem-resistant $P$. aeruginosa strains were recovered (108 patients) and in which ceftazidime-resistant strains were recovered (56 patients) were selected during the years 1999-2002. Case-control studies showed that independent risk factors associated with imipenem resistance in their hospital were transfer from another hospital (perhaps a marker for longer time at risk), receipt of hemodialysis, and use of imipenem, amikacin, or vancomycin. Only transfer from another hospital and amikacin use were independent risk factors for recovery of ceftazidimeresistant $P$. aeruginosa; ceftazidime use was not.

The setting of the study by Lautenbach et al. ${ }^{3}$ was a $625-$ bed tertiary care hospital in Philadelphia, Pennsylvania. Cases in which imipenem-resistant $P$. aeruginosa strains were recovered (142 patients) were identified during the years 19992000. Results of a case-control study showed that fluoroquinolone use, and not imipenem use, was an independent risk factor for recovery of an imipenem-resistant strain.

Why was imipenem use a risk factor in one study and not in the other? Both were case-control studies that evaluated imipenem resistance in $P$. aeruginosa at large teaching hospitals. There was a similar distribution of anatomical sites from which the clinical specimens were isolated and a similar amount of intensive care unit exposure in both studies.

Recent studies have discussed the importance of the method used to select control subjects in case-control studies of antibiotic resistance. ${ }^{5,6}$ In the study by Lautenbach et al., ${ }^{3}$ the question being asked was "Among clinical isolates of $P$. aeruginosa, what are the risk factors associated with imipenem resistance in a given isolate?" Thus, patients colonized or infected with imipenem-resistant $P$. aeruginosa were classified as case patients and patients colonized or infected with imipenem-susceptible $P$. aeruginosa were classified as control subjects. The study by Fortaleza et al. ${ }^{4}$ was designed to evaluate risk factors associated with the recovery of imipenemresistant and ceftazidime-resistant $P$. aeruginosa isolates from patients admitted to the authors' hospital. Case patients were those from whom imipenem-resistant or ceftazidime-resistant strains of $P$. aeruginosa were recovered, and control subjects matched to case patients were selected from the patients admitted to the same ward. The questions asked were different, and thus the procedures for selection of control subjects were different.

Harris et al. $^{5}$ have reviewed the methodologies for casecontrol studies of antibiotic resistance that may lead to varying results with respect to risk factors. Both Lautenbach et al. ${ }^{3}$ and Fortaleza et al. ${ }^{4}$ acknowledged the impact of methodology and chose control subjects on the basis of their intended question. The novel case-case-control study design described by Kaye et al. ${ }^{6}$ addresses some limitations in study design and has been used to study risk factors for colonization or infection with imipenem-resistant $P$. aeruginosa among hospitalized patients. ${ }^{7}$ Findings of the study by Kaye et al. ${ }^{6}$ showed that receipt of imipenem, piperacillin-tazobactam, vancomycin, and/or aminoglycosides were risk factors for isolation of imipenem-resistant $P$. aeruginosa. The odds ratio for the risk of isolation of imipenem-resistant $P$. aeruginosa, how-

From the Department of Medicine, University of Texas Health Science Center at San Antonio, and the Medical Service, South Texas Veterans Health Care System, San Antonio, Texas.

Received July 4, 2006; accepted July 4, 2006; electronically published August 24, 2006.

Infect Control Hosp Epidemiol 2006; 27:889-892

(C) 2006 by The Society for Healthcare Epidemiology of America. All rights reserved. 0899-823X/2006/2709-0001\$15.00. 
ever, was lower than that found in a previous study, ${ }^{8}$ perhaps because of the difference in the method of selection for control subjects. It is also of interest that both Harris et al. ${ }^{7}$ and Fortaleza et al. ${ }^{4}$ found vancomycin use to be a risk factor for recovery of imipenem-resistant $P$. aeruginosa. As Harris et al. ${ }^{7}$ suggest, this may be because of the deleterious effect of a decrease in the population of endogenous gram-positive flora, which allows $P$. aeruginosa to proliferate.

In addition, as Fortaleza et al. ${ }^{4}$ point out, other factors in addition to case-control study methodology can lead to different results in studies of risk factors for recovery of drugresistant organisms. These include the geographic location where strains are isolated, since mechanisms of resistance may differ between locales, and variation in prescribing patterns. For instance, Fortaleza et al. $^{4}$ refer to the dissemination of metallo- $\beta$-lactamase-producing $P$. aeruginosa in Brazilian hospitals. ${ }^{y}$ Yet, in the United States, metallo- $\beta$-lactamases have not been commonly detected in $P$. aeruginosa, so the mechanisms of imipenem resistance may be different between Philadelphia and Campinas, Brazil.

The study by Lautenbach et al. ${ }^{3}$ finds fluoroquinolone use, not imipenem use, to be a risk factor for emergence of imipenem resistance in $P$. aeruginosa. Why would fluoroquinolones select for imipenem-resistant $P$. aeruginosa? Mechanisms of resistance to fluoroquinolones include not only alterations in DNA gyrase but reduction or alteration in outer-membrane proteins and efflux as well. As Lautenbach et al. ${ }^{3}$ discuss, in vitro studies have suggested that emergence of imipenem resistance after treatment with fluoroquinolones has occurred. This is plausible, since both agents enter the cell via outer-membrane proteins and can be expelled from the cell by efflux systems. ${ }^{10}$ Neither Lautenbach et al..$^{3}$ nor Fortaleza et al. ${ }^{4}$ evaluated the mechanism of imipenem resistance in the strains they isolated, but since imipenem metallo- $\beta$-lactamases are common in Brazil and not in the United States, and since fluoroquinolone use can select for imipenem resistance mediated by change in outer-membrane proteins or efflux systems, the mechanisms of imipenem resistance may well be different in these 2 geographic areas, resulting in different antibiotic selection risk factors.

Use of multiple antibiotics, alone or in combination, were risk factors for infection with imipenem-resistant $P$. aeruginosa in both studies, although Lautenbach et al. ${ }^{3}$ found that use of antibiotics other than imipenem did not remain as independent risk factors. A salient point from both studies is that the restriction of imipenem use alone should not be the only strategy for controlling emergence of resistance. The use of multiple antibiotics should be judicious since this also contributes to resistance. Although it is important for empirical therapy to be appropriately broad for seriously ill patients, "it is just as important to de-escalate therapy to culture-directed therapy once a pathogen is isolated, or to narrow or discontinue empirical therapy once a suspected drug-resistant pathogen or infection is ruled out.

The study of Lautenbach et $a .^{3}$ showed that infection with imipenem-resistant $P$. aeruginosa is associated with increased hospital mortality, and other studies have also showed this. ${ }^{12,13}$ In this same issue, Gomes et al. ${ }^{14}$ report from a 2,000-bed tertiary care hospital in São Paolo, Brazil, that cephalosporin resistance in $K$. pneumoniae is not associated with increased mortality. Why the different conclusions with regard to antibiotic resistance and mortality? First, these are different pathogens. The virulence of $P$. aeruginosa, particularly in serious infections, is well-known. ${ }^{12,13,15}$ Although mortality associated with serious infection due to cephalosporin-resistant, extended-spectrum $\beta$-lactamase (ESBL)-producing $K$. pneumoniae may be lower than that associated with $P$. aeruginosa infection, nonurinary-tract $K$. pneumoniae infections have also been associated with significant mortality. ${ }^{16}$ In addition, previous studies have suggested that mortality may be higher among patients infected with ESBL-producing $K$. pneumoniae, especially those who do not receive appropriate initial therapy. ${ }^{17}$ Inadequate initial therapy has been shown in a number of studies to be a significant predictor of hospital mortality in seriously ill patients, ${ }^{11,15,16,18}$ although some studies have not found this correlation. ${ }^{19,20}$ For ESBL-producing $K$. pneumoniae, in particular, the impact of inadequate initial therapy varies by site of infection. ${ }^{16,21}$ In the study of Gomes et al., ${ }^{14}$ the top 3 types of infection were the urinary tract infection (39\%), the primary bloodstream infection $(16 \%)$, and the lower respiratory tract infection (13\%). The study of Gomes et al. ${ }^{14}$ appears to differ from a recent study by Paterson et al., ${ }^{21}$ which was a multicenter global study of ESBLproducing K. pneumoniae or Escherichia coli bacteremias only. It evaluated mortality and clinical response rates among patients treated with cephalosporins alone, compared with patients treated with a carbapenem or combination therapy. The study of Paterson et al..$^{21}$ showed a high treatment-failure rate and higher mortality among patients with these infections who were treated with cephalosporins alone. Paterson et al. ${ }^{21}$ looked at cases of bacteremia only, and Gomes et al. ${ }^{14}$ looked at cases of infection identified from specimens from all anatomical sites. Even though Gomes et al. ${ }^{14}$ did a separate evaluation of infections identified from blood and specimens from sterile sites that showed the same result, this was a small sample.

Why the differences in the association between clinical failure and inadequate therapy in these 2 studies? Both are relatively small studies and thus are limited in statistical power. As Gomes et al. ${ }^{14}$ point out, in their study cephalosporin doses could not be well studied, and dosing may be critical for treatment efficacy. Also, the mechanism of cephalosporin resistance was not evaluated by Gomes et al., ${ }^{14}$ whereas Paterson et al. $^{21}$ confirmed the presence of ESBLs. In addition, types of ESBLs vary by geographic location, and the type of ESBL can make a difference in the response to therapy, since some antibiotics are better substrates for certain ESBLs than others. ${ }^{22}$ The specific cephalosporins used, predominantly or individually, are not described in the study of Gomes et al., ${ }^{14}$ thus limiting evaluation of this factor. Finally, Paterson et al. ${ }^{21}$ 
looked at infections from multiple geographic sites; the study of Gomes et al. ${ }^{14}$ was limited to one hospital in São Paolo, Brazil.

The study of Gomes et al. ${ }^{14}$ does suggest an important point for the hospital under study, though, and potentially for other similar hospitals. The results from their hospital mean that broader-spectrum agents or regimens, such as carbapenems or combination therapy, may not need to be used widely for empirical therapy in their hospital, despite the prevalence of cephalosporin resistance. This could prevent unnecessary antibiotic use and save costs in a setting where such considerations are particularly important.

Clonality of strains was not evaluated in any of these studies, but transmission of multidrug-resistant gram-negative pathogens is known to play an important role in the emergence of this problem in hospitals. The study by Gunale et al., ${ }^{23}$ also in this issue of the journal, expands the investigation of the known transmission of gram-negative pathogens on the hands of health care workers. Ten genetically distinct cephalosporin-resistant strains ( 5 transmitted and 5 sporadic) of Enterobacter cloacae and Pantoea agglomerans (formerly Enterobacter agglomerans) were inoculated on the fingertips of 10 healthy volunteers who had no skin conditions and short fingernails. Fingertips were sampled at time 0 and at 15,30 , and 45 minutes to determine the survival rate of the pathogens. There was no difference in survival rates between the transmitted strains and the sporadic strains, suggesting that there are no significant intrinsic differences between these types of strains with respect to their survival rate on fingers. There were, however, differences between test subjects. Seven of the 10 strains survived best on one person, who had a consistently higher bacterial count than the other test subjects. There were substantial decreases in the viable bacteria count after the first 15 minutes, but in some instances the bacteria survived for up to 45 minutes.

The study of Gunale et al..$^{23}$ is further evidence of the importance of transient hand carriage, and individuals' predisposition for it, in the hospital setting, and reinforces the importance of hand antisepsis as a cornerstone for preventing the spread of resistant organisms. Although neither Lautenbach et al., ${ }^{3}$ Fortaleza et al., ${ }^{4}$ nor Gomes et al. ${ }^{14}$ evaluated clonality among the drug-resistant strains they studied, it is likely that some of the cases of infection represented isolates spread between patients. This mechanism continues to be an important one for the spread of resistance among gram-negative pathogens.

So, what can we conclude from these studies about measures that should be enacted to prevent the emergence of resistance among gram-negative pathogens? Overall mortality from infection with gram-negative pathogens, antibiotic-resistant or not, remains high. Mortality due to infection with multidrug-resistant $P$. aeruginosa is of the most concern. Restriction of the use of individual antibiotics alone is unlikely to be successful in the prevention of emergence of imipenem resistance. The use of fluoroquinolones and multiple anti- biotics also contributes. The determination of local patterns and mechanisms of resistance is key in determining which antibiotic-use measures will work in one's own institution. Basic measures, such as hand antisepsis, are crucial and need further implementation and reinforcement. The lack of alternative agents on the horizon that are active against gramnegative bacteria makes our efforts at controlling emergence of resistance all the more imperative.

Address reprint requests to Jan E. Patterson, MD, Chief of Medicine, South Texas Veterans Health Care System, San Antonio, TX 78229 (pattersonj @uthscsa.edu).

\section{REFERENCES}

1. Gardiner DF, Scholand SJ, Babinchak. Mortality and gram-negative rod bacteremia in the intensive care unit. J Hosp Infect 2006; 62:453-457.

2. Friedman G, Sliva E, Vincent JL. Has the mortality of septic shock changed with time? Crit Care Med 1998; 26:2078-2080.

3. Lautenbach E, Weiner MG, Nachamkin I, Bilker WB, Sheridan A, Fishman NO. Imipenem resistance among Pseudomonas aeruginosa isolates: risk factors for infection and impact of resistance on clinical and economic outcomes. Infect Control Hosp Epidemiol 2006; 27:893-900 (in this issue).

4. Fortaleza CMCB, Freire MP, Moreira Filho DC, Carvalho Ramos M. Risk factors for recovery of imipenem- or ceftazidime-resistant Pseudomonas aeruginosa among patients admitted to a teaching hospital in Brazil. Infect Control Hosp Epidemiol 2006; 27:901-906 (in this issue).

5. Harris AD, Karchmer TB, Carmeli Y, Samore MH. Methodological principles of case-control studies that analyzed risk factors for antibiotic resistance: a systematic review. Clin Infect Dis 2001; 32:1055-1061.

6. Kaye KS, Harris AD, Samore M, Carmeli Y. The case-case-control study design: addressing the limitation of risk factor studies for antimicrobial resistance. Infect Control Hosp Epidemiol 2005; 26:346-351.

7. Harris AD, Smith D, Johnson JA, Bradham DD, Roghmann MC. Risk factors for imipenem-resistant Pseudomonas aeruginosa among hospitalized patients. Clin Infect Dis 2002; 34:340-345.

8. Troillet N, Samore MH, Carmeli Y. Imipenem-resistant Pseudomonas aeruginosa: risk factors and antibiotic susceptibility patterns. Clin Infect Dis 1997; 25:1094-1098.

9. Gales AC, Menezes LC, Silbert S, Sader HS. Dissemination in distinct Brazilian regions of an epidemic carbapenem-resistant Pseudomonas aeruginosa producing SPM metallo-beta-lactamase. J Antimicrob Chemother 2003; 52:699-702.

10. Aubert G, Pozzetto B, Dorche G. Emergence of quinolone-imipenem cross-resistance in Pseudomonas aeruginosa after fluoroquinolone therapy. J Antimicrob Chemother 1992; 29:307-312.

11. Kollef MH, Sherman G, Ward S, Fraser VJ. Inadequate antimicrobial treatment of infections: a risk factor for hospital mortality among critically ill patients. Chest 1999; 115:462-474.

12. Marra AR, Pereira CAP, Gales AC, et al. Bloodstream infections with metallo-beta-lactamase-producing Pseudomonas aeruginosa: epidemiology, microbiology, and clinical outcomes. Antimicrob Agents Chemother 2006; 50:388-390.

13. Kang CI, Kim SH, Park WB, et al. Bloodstream infections caused by antibiotic-resistant gram-negative bacilli: risk factors for mortality and impact of inappropriate initial antimicrobial therapy on outcome. Antimicrob Agents Chemother 2005; 49:760-766.

14. Gomes CC, Vormittag E, Santos CR, Levin AS. Nosocomial infection with cephalosporin-resistant Klebsiella pneumoniae is not associated with increased mortality. Infect Control Hosp Epidemiol 2006; 27:907-912 (in this issue). 
15. Ibrahim EH, Sherman G, Ward S, Fraser VJ, Kollef MH. The influence of inadequate antimicrobial treatment of bloodstream infections on patient outcomes in the ICU setting. Chest 2000; 118:146-155.

16. Hyle EP, Lipworth AD, Zaoutis TE, Nachamkin I, Bilker WB, Lautenbach E. Impact of inadequate initial antimicrobial therapy on mortality in infections due to extended-spectrum beta-lactamase-producing Enterobacteriaceae. Arch Intern Med 2005; 165:1375-1380.

17. Ramphal R, Ambrose PG. Extended-spectrum beta-lactamases and clinical outcomes: current data. Clin Infect Dis 2006; 42(Suppl 4):S164-S172.

18. Harbarth S, Garbino J, Pugin J, Romand JA, Lew D, Pittet D. Inappropriate initial antimicrobial therapy and its effect on survival in a clinical trial of immunomodulating therapy for severe sepsis. Am J Med 2003; 115:529-535.

19. Bates DW, Pruess KE, Lee TH. How bad are bacteremia and sepsis?
Outcomes in a cohort with suspected bacteremia. Arch Intern Med 1995; 155:593-598.

20. Dupont $\mathrm{H}$, Montravers $\mathrm{P}$, Gauzit $\mathrm{R}$, et al. Outcome of postoperative pneumonia in the EOLE study. Intensive Care Med 2003; 29:179-188.

21. Paterson DL, Ko WC, Von Gottberg A, et al. Outcome of cephalosporin treatment serious infections due to apparently susceptible organisms producing extended-spectrum beta-lactamases: implications for the clinical microbiology laboratory. J Clin Microbiol 2001; 39:2206-2212.

22. Andes D, Craig WA. Treatment of infections with ESBL-producing organisms: pharmacokinetic and pharmacodynamic considerations. Clin Microbiol Infect 2005; 11(Suppl 6):10-17.

23. Gunale A, von Baum P, Wendt C. Survival of cephalosporin-resistant Enterobacteriaceae on fingers. Infect Control Hosp Epidemiol 2006; 27: 974-977 (in this issue). 